\title{
On Deriving Tidal Volume From Electrocardiogram During Maximal Effort Test
}

\author{
Javier Milagro ${ }^{1,2}$, David Hernando ${ }^{1,2}$, Jesús Lázaro ${ }^{1,2,3}$, José A Casajús ${ }^{4,5}$, \\ Nuria Garatachea ${ }^{4,5}$, Eduardo Gil ${ }^{1,2}$, Raquel Bailón ${ }^{1,2}$ \\ ${ }^{1}$ BSICoS Group, Aragón Institute of Engineering Research (I3A), IIS Aragón, University of \\ Zaragoza, Zaragoza, Spain \\ ${ }^{2}$ CIBER - Bioingeniería, Biomateriales y Nanomedicina (CIBER-BBN), Madrid, Spain \\ ${ }^{3}$ Department of Biomedical Engineering, University of Connecticut, Storrs CT, USA \\ ${ }^{4}$ GENUD Research Group, Faculty of Health and Sport Sciences, IA2, IIS Aragón, University of \\ Zaragoza, Zaragoza, Spain \\ ${ }^{5}$ CIBER - Fisiopatología de la Obesidad y Nutrición (CIBERObn), Madrid, Spain
}

\begin{abstract}
Electrocardiogram-derived respiration (EDR) is a well known method for obtaining respiratory signal estimations only from electrocardiogram (ECG). It has been extensively employed for deriving respiratory rate, whereas much less results concerning tidal volume (TV) estimation have been reported in the literature. In this work, a method for estimating TV from ECG during a maximal effort treadmill test is presented. It is based on the R-S amplitude series and a calibration process of a linear model. The test was divided in stages, and lowest estimation error was obtained for lead V4, with a median relative error as low as $7 \%$ in the best-performing stage and lower than $14 \%$ in most of them, suggesting that TV can be estimated from the ECG.
\end{abstract}

\section{Introduction}

Electrocardiogram (ECG) has a wide range of clinical applications as it contains large information about heart condition. Furthermore, some respiration-related modulations are present in the ECG, so respiratory information can be extracted from it [1-4]. One of those modulations is the so called respiratory sinus arrhythmia (RSA), which reflects as a tachycardia during inspiration followed by a bradycardia during expiration. Moreover, ECG morphology is modulated by electrodes movement with respect to the heart, as well as impedance changes in the thorax due to the amount of air in the lungs. This effect leads to an increased electrical impedance of the thorax during inspiration, which is reflected in the ECG as amplitude and shape variations. Several techniques to esti- mate respiratory rate from ECG signals have been proposed in the literature. Some of them exploit different morphological features such as variations in the R or Rto-S waves amplitude [1], the QRS-complex area [2], the QRS-complex slopes [3] or vectocardiogram rotations [4]. However, only little research concerning the estimation of tidal volume (TV) has been published. To the best of our knowledge, previous studies focused on noninvasive TV estimation have mostly considered ECG-unrelated techniques, such as inductive [5] or opto-electronic plethysmography [6], traqueal sounds [7] or even image acquisition using a smarthpone camera [8]. However, proportionality between ECG-derived respiration (EDR) and TV was reported in [2], whereas in [9] they conducted a conceptual study for estimating TV from ECG or intra-cardiac signals [9] in mechanically ventilated swines, hence in a controlled environment. In this work, a method for TV estimation from ECG is proposed. The method is based on R-S amplitude series and it is evaluated with signals recorded during a maximal effort treadmill test, which remains a noisy and highly non-stationary environment.

\section{Materials and Methods}

\subsection{Database}

25 apparently healthy male volunteers $(33.4 \pm 5.2$ years) were recruited. All of them were active and participated in aerobic training at least 3 days per week. They performed a maximal treadmill (Quasar MED LT h/p Cosmos) effort test, which was divided in 3 stages: a 5-minute resting stage (while seated), an exercise stage and a recovery stage. During the exercise stage, the subjects started to run at $8 \mathrm{~km} / \mathrm{h}$ and the speed was gradually increased 
at $1 \mathrm{~km} / \mathrm{h}$ per minute, until the subjects stopped due to volitional exhaustion. Afterwards, recovery stage started, and subjects were requested to keep running at $8 \mathrm{~km} / \mathrm{h}$ for 3-5 additional minutes. Multilead ECG was acquired at $1000 \mathrm{~Hz}$ using a high resolution holter (Mortara 48hour H12+, Mortara Instrument, Milwaukee,Wisconsin). Leads I, II, III, aVL, aVR, aVF, V4, V5 and V6 were obtained. Minute ventilation $\left(V_{\mathrm{E}}\right)$ and respiratory rate $\left(f_{\text {resp }}\right)$ were recorded breath-by-breath with an Oxycon Pro device (Jaeger/Viasys, Germany). Recordings were performed at University of Zaragoza, and written informed consent was received from all the participants.

\subsection{Preprocessing}

Beat detection and delineation was performed in each ECG lead by using the wavelet-based method described in [10]. For simplicity, temporal series will be displayed in bold type. From beat time occurrence, instantaneous heart rate (HR), $\boldsymbol{x}_{\mathrm{HR}}$, was obtained. Breath-to-breath TV $\left(\boldsymbol{V}_{\mathrm{T}}\right)$ was calculated as $\boldsymbol{V}_{\mathrm{E}} / \boldsymbol{f}_{\text {resp }}$. Afterwards, $\boldsymbol{x}_{\mathrm{HR}}$ and $\boldsymbol{V}_{\mathrm{T}}$ were synchronized (a breath-to-breath HR measurement provided by the Oxycon Pro device was used for this purpose) and smoothed with a 10-sample median filter.

Each R-to-S amplitude series, $\boldsymbol{x}_{\mathrm{R}-\mathrm{S}}$, was used as EDR signal. $\boldsymbol{x}_{\mathrm{R}-\mathrm{S}}$ was first low-pass filtered at $1 \mathrm{~Hz}$ to discard high frequencies unrelated with respiration, and the result of this filtering was referred to as $\tilde{\boldsymbol{x}}_{\mathrm{R}-\mathrm{s}}$. Time occurrence of the peaks in $\tilde{\boldsymbol{x}}_{\mathrm{R}-\mathrm{S}}$ should be related with expiration, as higher EDR amplitude is expected when electrodes are closer to the heart. We detected those peaks and the peak amplitude series was filtered with a 10-sample median filter. The outcome of this filtering process, $\tilde{\boldsymbol{x}}_{p k}$, was resampled at the time instants when volume information was available and the resulting signal was referred to as $\boldsymbol{x}$. An example of this preprocessing stage is displayed in Fig. 1.

\subsection{Tidal Volume Modeling}

The effort test was divided into 5 different stages: rest $\left(I_{\text {rest }}\right), 0-60 \%\left(I_{0-60}\right), 60-80 \%\left(I_{60-80}\right)$ and $80-100 \%\left(I_{80-100}\right)$ of maximum HR, and recovery $\left(I_{\text {recov }}\right)$ stage. $I_{\text {rest }}$ was set as the time from signal acquisition start until 30 seconds before exercise onset (to avoid the transition between rest and the start of the exercise stage) and $I_{\text {recov }}$ as the time from 30 seconds after maximum HR was achieved (exercise offset) until the end of the recording. The segmentation of the other 3 stages was performed automatically from the $\boldsymbol{x}_{\mathrm{HR}}$ signal (Fig. 2 a)) considering the mean HR at $I_{\text {rest }}$ as $0 \%$, and the maximum reached $\mathrm{HR}$ as $100 \%$.

In each of the previous stages, $\boldsymbol{x}$ was fitted to $\boldsymbol{V}_{\mathrm{T}}$ with a simple first-order linear model:

$$
\hat{\boldsymbol{V}}_{\mathrm{T}}^{I}=\theta_{0}^{I}+\boldsymbol{x}^{I} \theta_{1}^{I},
$$
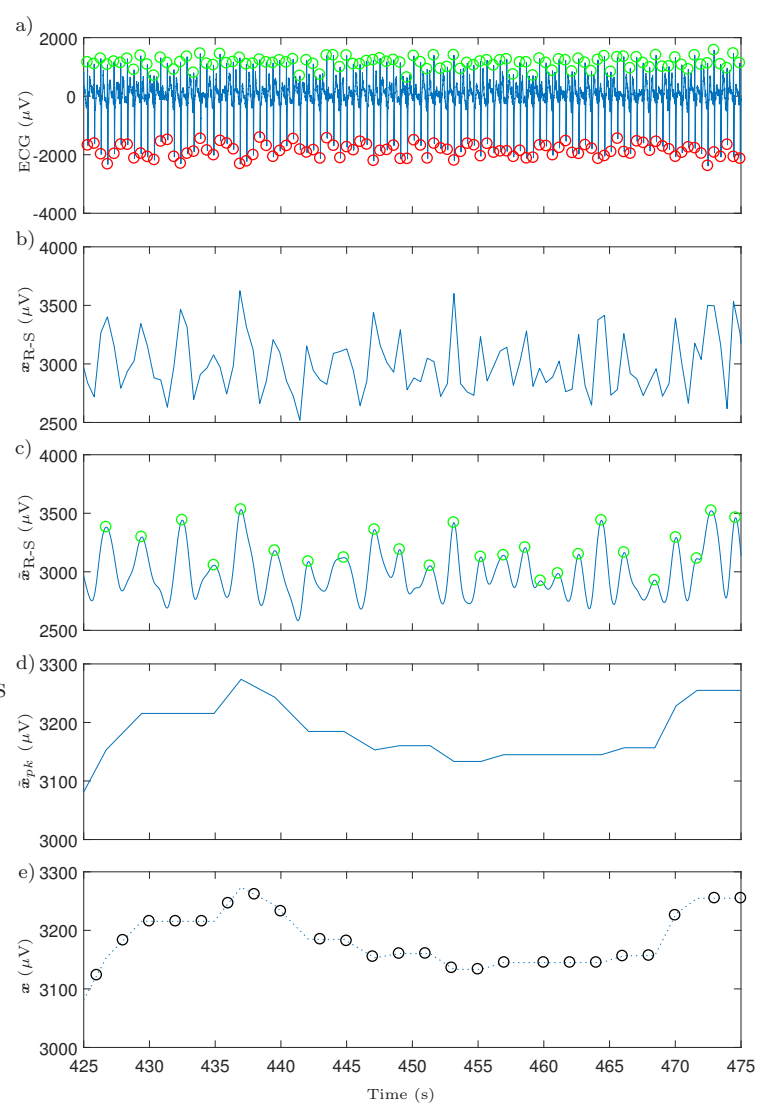

Figure 1. In a), $\mathrm{R}$ and $\mathrm{S}$ waves (green and red circles respectively) were detected in the ECG. R-S amplitude series generated from this detections, $\boldsymbol{x}_{\mathrm{R}-\mathrm{S}}$, is displayed in $\mathrm{b}$ ). In c), $\boldsymbol{x}_{\mathrm{R}-\mathrm{S}}$ was low-pass filtered at $1 \mathrm{~Hz}$, and the peaks corresponding to maximum $\mathrm{R}-\mathrm{S}$ amplitude are marked with green circles. The peak amplitude series was filtered with a 10 -sample median filter to generate $\tilde{\boldsymbol{x}}_{p k}$, as shown in d). Finally, $\tilde{\boldsymbol{x}}_{p k}$ was re-sampled at the time instants when volume information was available (labeled with black circles in e)) in order to obtain $\boldsymbol{x}$.

where superindex $I$ denotes the considered stage, $\theta_{0}^{I}$ and $\theta_{1}^{I}$ are the offset and slope of the liner model respectively, and $\boldsymbol{x}^{I}$ is the vector formed by the samples of $\boldsymbol{x}$ contained in stage $I$. For the calibration, only $20 \%$ of the samples of the previous interval and $30 \%$ of the samples of the interval to predict were considered. Afterwards, the remaining $70 \%$ were estimated from the model. This calibration process was repeated for each subject, stage and lead. An example of this estimation is displayed in Fig. 2 b). In a second approach, we proposed a multi-lead methodology including information of all the leads. For this purpose, a principal component analysis (PCA) was applied to merge the $\boldsymbol{x}_{\mathrm{R}-\mathrm{S}}$ of all the leads in a single series. The first PCA component, $\boldsymbol{x}_{\mathrm{PCA}}$, was used as the EDR signal in this approach. Then, the same processing described in Section 2.2 was applied. Finally, it was fitted to $\boldsymbol{V}_{\mathrm{T}}$ as in Eq. (1). 
Table 1. Absolute $\left(\epsilon_{a}\right)$ and relative $\left(\epsilon_{r}\right)$ estimation error for each of the five stages. The data is presented as median of medians (median of IQRs). Values corresponding to leads V4 and aVF, and to the multi-lead approach are shown.

\begin{tabular}{ccccccccc}
\hline \hline & \multicolumn{2}{c}{$\mathrm{V} 4$} & & \multicolumn{2}{c}{$\mathrm{aVF}$} & & \multicolumn{2}{c}{ PCA } \\
\cline { 2 - 3 } \cline { 7 - 8 } & $\epsilon_{a}$ (liters) & $\epsilon_{r}(\%)$ & & $\epsilon_{a}$ (liters) & $\epsilon_{r}(\%)$ & & $\epsilon_{a}$ (liters) & $\epsilon_{r}(\%)$ \\
\hline$I_{\text {rest }}$ & $0.10(0.11)$ & $11.40(14.46)$ & & $0.10(0.11)$ & $11.73(14.90)$ & & $0.11(0.11)$ & $11.89(14.43)$ \\
$I_{0-60}$ & $0.21(0.22)$ & $13.08(9.55)$ & & $0.84(0.31)$ & $46.10(13.41)$ & & $0.30(0.22)$ & $20.03(8.28)$ \\
$I_{60-80}$ & $0.21(0.17)$ & $8.67(6.83)$ & & $0.25(0.20)$ & $11.58(7.83)$ & & $0.16(0.15)$ & $5.62(5.91)$ \\
$I_{80-100}$ & $0.23(0.17)$ & $7.00(5.93)$ & & $0.20(0.18)$ & $8.09(5.41)$ & & $0.21(0.19)$ & $7.66(6.74)$ \\
$I_{\text {recov }}$ & $0.44(0.25)$ & $17.67(15.49)$ & & $0.38(0.25)$ & $16.67(11.54)$ & & $0.46(0.30)$ & $18.78(12.09)$ \\
\hline \hline
\end{tabular}
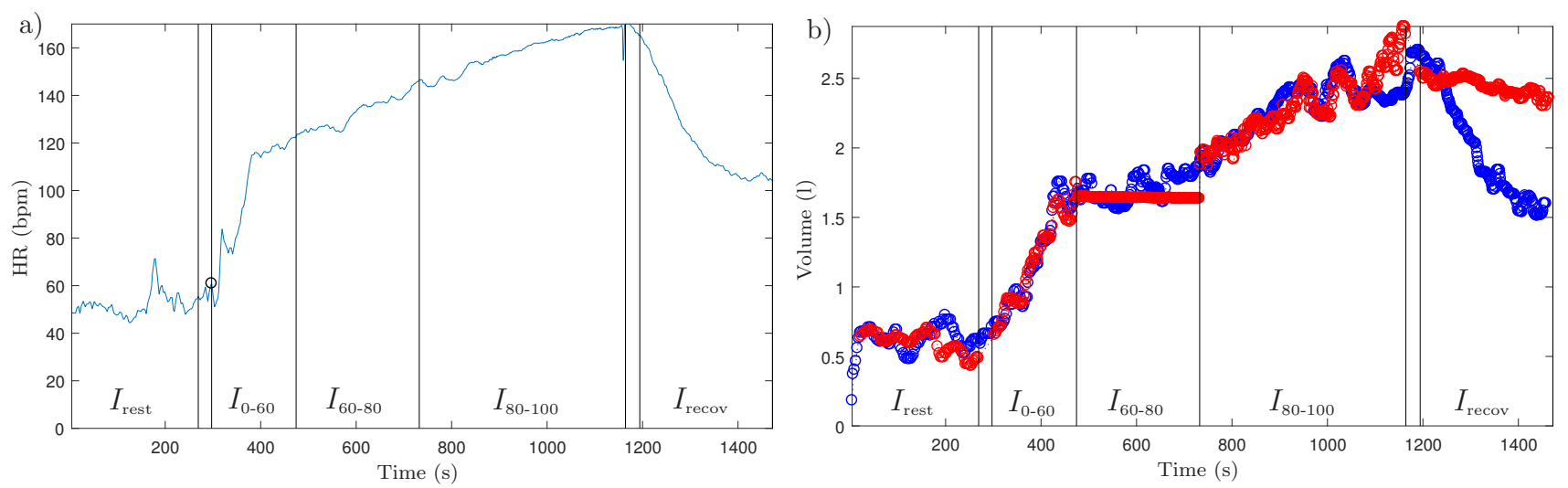

Figure 2. Automatic segmentation of the maximal effort test based on HR is shown in a). $I_{\text {rest }}$ is set from the beginning of the recording until 30-seconds prior to exercise onset (black circle), and $I_{\text {recov }}$ goes from the time when maximum HR is reached to the end of the recording. $I_{0-60}, I_{60-80}$ and $I_{80-100}$ are automatically defined according to HR, considering the mean HR at $I_{\text {rest }}$ as $0 \%$ and the maximum reached HR as $100 \%$. In b), volume estimation corresponding to the same subject than in a) is shown. $\boldsymbol{V}_{\mathrm{T}}$ is represented with blue circles, whereas $\hat{\boldsymbol{V}}_{\mathrm{T}}$ is represented with red circles. Despite all the points in each stage are depicted, only the last $70 \%$ of them were considered for the fitting error results displayed in Table 1 .

\subsection{Performance Measurement}

In order to quantify the accuracy of the estimation, absolute $\left(\epsilon_{a}\right)$ and relative $\left(\epsilon_{r}\right)$ error were calculated for each stage, lead and subject. Inter-subject median of medians and median of interquartile ranges (IQRs) of $\epsilon_{a}$ and $\epsilon_{r}$ were computed for each stage and lead.

\section{Results}

Median estimation error obtained for each stage is displayed in Table 1. 7 subjects had to be discarded due to bad signal quality or missing ECG or TV signals. Although similar, different results were achieved depending on the considered lead, so results for leads V4 and aVF were considered here. Lowest estimation relative error was achieved for $I_{80-100}$ in V4 and aVF, and in $I_{60-80}$ in PCA, being the latter as low as $5.62 \%$. Relative errors lower than 9 and $12 \%$ were achieved in $I_{60-80}$ and $I_{\text {rest }}$ in all the cases (except for aVF, with a relative error of $11.58 \%$ in $I_{60-80}$ ). In $I_{0.60}$, error was again kept below $14 \%$ when considering
V4, whereas it increased for the other options (especially for aVF, reaching a median relative error of $46.10 \%$ ). Finally, highest errors for V4 and PCA were found in $I_{\text {recov }}$ (although still lower than 19\%). In aVF, a median relative error of $16.67 \%$ was achieved for the latter stage.

\section{Discussion}

In this work, a simple approach for estimating TV from ECG signals under rest and non-stationary conditions has been proposed. The wide variations in TV (less than 1 liter/breath during relaxed breathing and higher than 2.5 or 3 liters/breath at maximal HR) made it necessary to subdivide the test in different intervals, and automatic segmentation based on exercise onset and HR was performed. The use of the percentage of HR as an indicator for segmenting the test was due to the different behavior of TV variations according to HR (as displayed in Fig. 2).

As displayed in Table 1, different estimation errors were obtained for the different stages depending on the considered lead. E.g., V4 was the best performing lead in 
$I_{\text {rest }}, I_{0-60}$, and $I_{80-100}$, whereas aVF outperformed V4 in $I_{\text {recov }}$. These inter-lead differences could be produced by changes in the ECG modulation across the stages. In this way, reduced errors in V4 during the exercise stages might reflect that this lead is better capturing the effect that respiration exerts on the ECG, whilst in aVF the effect of respiration could be obscured by an increased effect of movement (due to electrode position). However, aVF led to the best results in $I_{\text {recov }}$, thus turning in a more adequate option in those cases (this improvement could be in fact caused by a reduction in the effect of movement). Nevertheless, the possibility that thorax expansion have a different effect depending on the relative position of the heart and the measurement leads remains an interesting possibility. If that was the case, different estimation error achieved among subjects could be related, apart from individual performance, physical condition or over-training, with differences in the leads position. Also the possibility of over-fitting in some cases must be considered.

Given the distinct behavior of the estimation in function of the considered lead, we proposed a combination of all the available leads through a PCA analysis. However, merging the information provided for all the leads did not resulted in any improvement with respect to the single-lead approach except in the case of $I_{60-80}$, as displayed in Table 1 .

A previous step to this work was proposed in [9], although this study presented some limitations. First, mechanical controlled ventilation is a very invasive procedure. Second, TV was subjected to controlled variations and kept constant during 90 seconds, thus creating a highly stationary scenario. Here, we have extended this concept to a very non-stationary situation, such as a maximum effort treadmill test, where TV is constantly varying. Lowest relative errors obtained in [9] were comprised between 7 and $10 \%$. With the proposed method, we achieved relative errors around $7 \%$ in the best-performing lead and stage.

Although low median error was achieved, we evaluated only one possible scenario per subject, so further work will be necessary in order to develop a subject-oriented model that allows us to estimate TV directly from ECG. This model would be really useful not only for non-invasive TV monitoring but also in the screening and monitoring of some respiration-related disorders, such as periodic breathing or sleep apnea.

\section{Conclusion}

A simple method to estimate TV from ECG has been proposed. This method obtained promising results both under rest and non-stationary conditions. The wide range of applications for monitoring and screening of noninvasive TV estimation encourages future work in this field.

\section{Acknowledgements}

This work was supported by grant BES-2015-073694 and project TIN2014-53567-R from Ministerio de Economía y Competitividad. Also by Government of Aragón and European Social Fund (EU) through BSI$\mathrm{CoS}$ group (T96) and by CIBER in Bioengineering, Biomaterials \& Nanomedicine (CIBER-BBN) through Instituto de Salud Carlos III. This project has received funding from the European Unions Framework Programme for Research and Innovation Horizon 2020 (2014-2020) under the Marie Skłodowska-Curie Grant Agreement No. 745755. The computation was performed by the ICTS NANBIOSIS, specifically by the High Performance Computing Unit of CIBER-BBN at University of Zaragoza.

\section{References}

[1] Mason C, Tarassenko L. Quantitative assessment of respiratory derivation algorithms. In Engineering in Medicine and Biology Society, 2001. Proceedings of the 23rd Annual International Conference of the IEEE, Volume 2. IEEE, 2001; 1998-2001.

[2] Moody GB, Mark RG, Zoccola A, Mantero S. Derivation of respiratory signals from multi-lead ECGs. Computers in Cardiology 1985;12(1985):113-116.

[3] Lázaro J, Alcaine A, Romero D, Gil E, Laguna P, Pueyo E, Bailón R. Electrocardiogram derived respiratory rate from QRS slopes and R-wave angle. Ann Biomed Eng 2014; 42(10):2072-2083.

[4] Bailón R, Sornmo L, Laguna P. A robust method for ECGbased estimation of the respiratory frequency during stress testing. IEEE Trans Biomed Eng 2006;53(7):1273-1285.

[5] Seppa VP, Viik J, Hyttinen J. Assessment of pulmonary flow using impedance pneumography. IEEE Trans Biomed Eng 2010;57(9):2277-2285.

[6] Reinaux CMA, Aliverti A, da Silva LGM, da Silva RJ, Gonçalves JN, Noronha JB, de Andrade AD, de Amorim Britto MC, et al. Tidal volume measurements in infants: Opto-electronic plethysmography versus pneumotachograph. Pediatr Pulmonol 2016;51(8):850-857.

[7] Reljin N, Reyes BA, Chon KH. Tidal volume estimation using the blanket fractal dimension of the tracheal sounds acquired by smartphone. Sensors 2015;15(5):9773-9790.

[8] Reyes BA, Reljin N, Kong Y, Nam Y, Chon KH. Tidal volume and instantaneous respiration rate estimation using a volumetric surrogate signal acquired via a smartphone camera. IEEE J Biomed Health Inform 2017;21(3):764-777.

[9] Sayadi O, Weiss EH, Merchant FM, Puppala D, Armoundas AA. An optimized method for estimating the tidal volume from intracardiac or body surface electrocardiographic signals: implications for estimating minute ventilation. Am J Physiol Heart Circ Physiol 2014;307(3):H426-H436.

[10] Martínez JP, Almeida R, Olmos S, Rocha AP, Laguna P. A wavelet-based ECG delineator: evaluation on standard databases. IEEE Trans Biomed Eng 2004;51(4):570-581.

Address for correspondence:

Javier Milagro

Dep. Ingeniería Electrónica y Comunicaciones. Universidad de Zaragoza, C/ María de Luna 1, L.3.07, 50018 Zaragoza, Spain milagro@unizar.es 